\title{
Topological piezoelectric effect and parity anomaly in nodal line semimetals
}

\author{
Taiki Matsushita, ${ }^{1}$ Satoshi Fujimoto, ${ }^{1}$ and Andreas P. Schnyder ${ }^{2}$ \\ ${ }^{1}$ Department of Materials Engineering Science, Osaka University, Toyonaka, Osaka 560-8531, Japan \\ ${ }^{2}$ Max-Planck-Institute for Solid State Research, Heisenbergstrasse 1, 70569 Stuttgart, Germany
}

(Received 26 February 2020; revised 2 August 2020; accepted 3 November 2020; published 3 December 2020)

\begin{abstract}
Lattice deformations act on the low-energy excitations of Dirac materials as effective axial vector fields. This allows one to directly detect quantum anomalies of Dirac materials via the response to axial gauge fields. We investigate the parity anomaly in Dirac nodal line semimetals induced by lattice vibrations and establish a topological piezoelectric effect; that is, periodic lattice deformations generate topological Hall currents that are transverse to the deformation field. The currents induced by this piezoelectric effect are dissipationless and their magnitude is completely determined by the length of the nodal ring, leading to a semiquantized transport coefficient. Our theoretical proposal can be experimentally realized in various nodal line semimetals, such as $\mathrm{CaAgP}$ and $\mathrm{Ca}_{3} \mathrm{P}_{2}$.
\end{abstract}

DOI: 10.1103/PhysRevResearch.2.043311

\section{INTRODUCTION}

Over the past few years a number of new types of topological semimetals have been discovered [1-7]. Among them are Weyl semimetals and Dirac semimetals with point nodes, around which the bands have linear dispersion in all directions. The low-energy physics of these point node semimetals is described by relativistic field theories with quantum anomalies, i.e., by quantum field theories that break symmetries of the classical action. For instance, two-dimensional Dirac materials, such as graphene, are described by quantum field theories with parity anomalies, which break space-time inversion symmetry $[8,9]$. The low-energy theories of Weyl semimetals, on the other hand, exhibit chiral anomalies, which violate conservation of axial charge [10-14]. The chiral anomaly in Weyl semimetals gives rise to numerous experimental phenomena [15-19], for example, negative magnetoresistance, which have been observed in recent experiments [19,20]. Lattice strain, which generates axial magnetic fields, can also be used to probe the chiral anomaly [21-34].

At the same time, recent research has focused on Dirac materials with line nodes [35-43]. These nodal-line semimetals (NLSMs) can be viewed as three-dimensional generalizations of graphene. They exhibit Dirac band crossings along a one-dimensional line in a three-dimensional Brillouin zone, with low-energy excitations that are linearly dispersing in the two directions perpendicular to the band-crossing line. Nodal-line semimetals possess a number of interesting properties, e.g., topological surface charges, drumhead surface states $[43,44]$, and quasitopological electromagnetic

Published by the American Physical Society under the terms of the Creative Commons Attribution 4.0 International license. Further distribution of this work must maintain attribution to the author(s) and the published article's title, journal citation, and DOI. responses $[45,46]$. The low-energy excitations around the nodal ring of these semimetals are described by one-parameter families of $(2+1)$-dimensional quantum field theories with parity anomalies [47]. That is, the electromagnetic responses of these nodal rings are given by Chern-Simons actions, which break parity symmetry. These Chern-Simons terms lead to transverse Hall effects, where electrons from opposite sides of the nodal ring flow to opposite surfaces, when an electric field is applied [47]. Unfortunately, due to time-reversal symmetry, the total current generated by the Chern-Simons action vanishes, once the sum over all momenta is taken. Therefore, the electric-field-induced Hall currents can only be measured by special devices that filter electrons based on their momenta [47].

In this paper we propose to use pseudo-electric-fields, induced by lattice vibrations, to probe the parity anomaly of NLSMs. As opposed to external electric fields, pseudoelectric-fields are axial, as they couple with opposite sign to electrons with opposite momenta. This permits us to directly probe the parity anomaly of NLSMs via the response to axial electric fields. We derive a low-energy description of NLSMs in the presence of strain and show that periodic lattice deformations generate a topological piezoelectric effect (TPEE), which originates from the parity anomaly. This piezoelectric effect manifests itself by dissipationless Hall currents that are transverse to the deformation field. We show that the TPEE can be interpreted as a polarization current and that it has a semiquantized transport coefficient, given by the length of the nodal ring. Furthermore, we discuss experimental considerations for the observation of the TPEE in the NLSM materials $\mathrm{CaAgP}$ and $\mathrm{Ca}_{3} \mathrm{P}_{2}$.

\section{MODEL}

First, we introduce a lattice model for a NLSM with a single nodal ring and discuss its topological properties. We consider the tight-binding Hamiltonian on the cubic 
lattice

$$
\begin{aligned}
H(\boldsymbol{p})= & t\left[2+\cos p_{0} a-\cos p_{x} a-\cos p_{y} a-\cos p_{z} a\right] \tau_{z} \\
& +v \sin p_{z} a \tau_{y}+\Delta \tau_{x},
\end{aligned}
$$

where $\tau_{i}(i=1,2,3)$ are Pauli matrices acting in orbital space. For simplicity, we assume $t, v, p_{0}>0$. To discuss the parity anomaly and the electric polarization, we have introduced a small parity-breaking term $\Delta \tau_{x}$. In the absence of $\Delta \tau_{x}$, the lattice Hamiltonian is parity-time $(\mathcal{P} \mathcal{T})$ symmetric with the $\mathcal{P} \mathcal{T}$ operator $\mathcal{P} \mathcal{T}=\tau_{z} K$. This tight-binding Hamiltonian describes the low-energy dispersion near the Fermi level of $\mathrm{CaAgP}$ and $\mathrm{Ca}_{3} \mathrm{P}_{2}[36,43]$. The symmetry-breaking term $\Delta \tau_{x}$ can be induced by applying uniaxial pressure or an electric field [47].

In the absence of $\Delta \tau_{x}$, Hamiltonian (1) exhibits a nodal ring within the $p_{z}=0$ plane, centered around $\Gamma$. This nodal ring is topologically protected by the $\mathbb{Z}_{2}$-invariant $v\left[S^{1}\right]$ [48],

$$
v\left[S^{1}\right]=\frac{1}{\pi} \sum_{\alpha \in \text { occ. states }} \oint_{S^{1}} d \boldsymbol{p} \cdot \boldsymbol{A}^{\alpha, \alpha}(\boldsymbol{p}) \bmod 2,
$$

where the integration path is along the closed loop $S^{1}$. Here $\mathcal{A}_{i}^{\alpha, \beta}(\boldsymbol{p})=i\left\langle u_{\alpha}(\boldsymbol{p})\left|\partial_{p_{i}}\right| u_{\beta}(\boldsymbol{p})\right\rangle$ and $\left|u_{\alpha}(\boldsymbol{p})\right\rangle$ are the Berry connection and the Bloch eigenstates of Eq. (1), respectively. In addition, $\mathcal{P} \mathcal{T}$ symmetry restricts Eq. (2) to the values $v\left[S^{1}\right]=$ 0,1 [49]. When the loop $S^{1}$ encircles the nodal ring, we obtain $v\left[S^{1}\right]=1$; otherwise $v\left[S^{1}\right]=0$.

The topological protection of the nodal ring is linked to a bulk electric polarization. To see this, let us decompose the three-dimensional Hamiltonian (1) into one-dimensional subsystems parametrized by the in-plane momenta $\boldsymbol{p}_{\perp}=$ $\left(p_{x}, p_{y}\right)$. The electric polarization of each subsystem is given by the Zak phase [50]

$$
P_{z}\left(\boldsymbol{p}_{\perp}\right)=\sum_{\alpha \in \text { occ. states }} \int_{-\pi}^{\pi} \frac{d p_{z}}{2 \pi} \mathcal{A}_{z}^{\alpha, \alpha}(\boldsymbol{p})=0, \frac{1}{2}
$$

and the total polarization is the summation of these phases over the in-plane momenta

$$
P_{z}=\int \frac{d \boldsymbol{p}_{\perp}}{(2 \pi)^{2}} P_{z}\left(\boldsymbol{p}_{\perp}\right)
$$

From Eqs. (2) and (3) we find that the Zak phase is $\frac{1}{2}$ for a region of in-plane momenta $\boldsymbol{p}_{\perp}$ that is bounded by the nodal ring. By the bulk-boundary correspondence [44], this leads to midgap surface states at the (001) face of the NLSM, whose fillings determine the surface charge. Due to $\mathcal{P} \mathcal{T}$ symmetry, the surface states at the top and bottom (001) faces are degenerate; thus the electric polarization is determined only up to a multiple of the elementary charge. To unambiguously determine the bulk polarization, it is necessary to include an infinitesimal $\mathcal{P} \mathcal{T}$-symmetry-breaking term $\Delta \tau_{x}$, which opens a bulk gap and removes the degeneracy of the midgap surface states. With the inclusion of $\Delta \tau_{x}$, we find that the bulk polarization is semiquantized and given by [46]

$$
P_{z}=\frac{S}{8 \pi^{2}} \operatorname{sgn}(\Delta)
$$

where $S$ is the area encircled by the nodal ring projected onto the surface Brillouin zone.

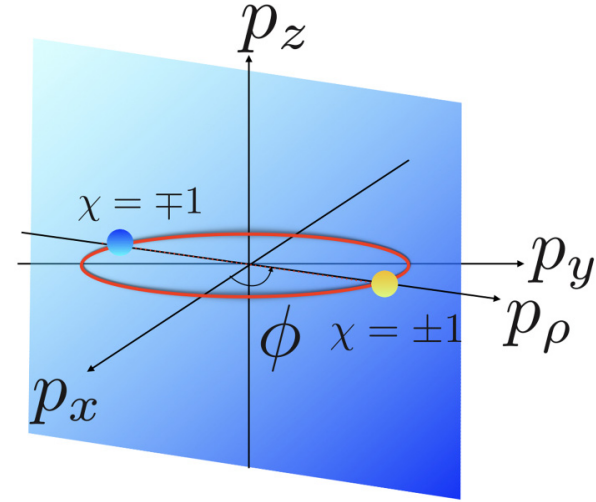

FIG. 1. Schematics of the nodal ring (red) within the $p_{z}=0$ plane. Using the cylindrical coordinates $\left(p_{\rho}, \phi, p_{z}\right)$, we decompose the NLSM into two-dimensional subsystems (blue) parametrized by $\phi$. Each subsystem contains two Dirac points with opposite sign of Berry curvature $\chi$.

\section{PARITY ANOMALY AND CHERN-SIMONS ACTION}

Next, we use a family of (2+1)-dimensional quantum field theories to derive the topological responses due to external and pseudo-electromagnetic-fields. For small p, Eq. (1) reduces to the low-energy continuum Hamiltonian

$$
H_{\mathrm{eff}}(\boldsymbol{p})=\frac{p^{2}-p_{0}^{2}}{2 m} \tau_{z}+\lambda p_{z} \tau_{y}+\Delta \tau_{x}
$$

where $1 / 2 m=t a^{2} / 2$ and $\lambda=v a$. Equation (6) has rotational symmetry around the $p_{z}$ axis. Thus, we introduce cylindrical coordinates $\left(p_{\rho}, \phi, p_{z}\right)$ with $p_{\rho} \in(-\infty, \infty)$ and $\phi \in$ $[0, \pi)$ (see Fig. 1). With these cylindrical coordinates, we can decompose the three-dimensional system into a family of two-dimensional subsystems labeled by the azimuth angle $\phi \in[0, \pi)$. Each of these subsystems contains two Dirac points that are related by time-reversal symmetry and have opposite sign of Berry curvature $\chi=\operatorname{sgn}(\Delta)= \pm 1$.

Each subsystem, labeled by $\phi$, is described by the $(2+1)$ dimensional quantum field theory

$$
\begin{aligned}
S^{\phi} & =\bigoplus_{\chi= \pm 1} S^{\phi, \chi}, \\
S^{\phi, \chi} & \left.=\int d^{2} x d t \bar{\psi}\left[i \chi \gamma^{\mu}\left(\partial_{\mu}+i A_{\mu}+i \chi A_{\mu}^{5}\right)+\Delta\right)\right] \psi,
\end{aligned}
$$

where $\psi$ is a two-component Dirac spinor, $\bar{\psi}=\psi^{\dagger} \gamma_{0}$, $\left\{\gamma_{\mu}, \gamma_{\nu}\right\}=\eta_{\mu \nu}$, and $\eta_{\mu \nu}=\operatorname{diag}(1,-1,-1)$. The Dirac spinors interact with the total gauge field $A_{\mu}^{\chi}=A_{\mu}+\chi A_{\mu}^{5}$, which contains both an external gauge field $A_{\mu}$ and an axial gauge field $A_{\mu}^{5}$. We note that the axial gauge field couples with opposite sign $\chi$ to the two Dirac points of the subsystem. The physical origin of $A_{\mu}^{5}$ due to lattice strain will be discussed later. Upon regularization [8], we obtain from Eq. (7) the parity-breaking Chern-Simons term

$$
S_{\mathrm{CS}}^{\phi, \chi}=\frac{\chi}{4 \pi} \int d^{2} x d t \epsilon^{\mu \nu \lambda} A_{\mu}^{\chi} \partial_{\nu} A_{\lambda}^{\chi},
$$

which is a manifestation of the parity anomaly. Varying the Chern-Simons action with respect to $A_{\mu}^{\chi}$ gives the anomalous 
transverse current

$$
j_{\mu}^{\phi, \chi}=-\frac{\delta S_{\mathrm{CS}}^{\phi, \chi}}{\delta A_{\mu}}=\frac{\chi}{4 \pi} \epsilon^{\mu \nu \lambda}\left(\partial_{\nu} A_{\lambda}+\chi \partial_{\nu} A_{\lambda}^{5}\right)
$$

for a single Dirac point with chirality $\chi$ in subsystem $\phi$. We observe that transverse currents induced by external electromagnetic fields cancel out, since contributions from opposite sides of the nodal ring have opposite sign $\chi= \pm 1$. Currents induced by axial gauge fields, however, do not cancel since they have the same sign everywhere along the nodal ring. This remarkable feature originates from the axial nature of the strain-induced gauge field $A_{\mu}^{5}$, which couples oppositely to Dirac fermions with opposite momenta.

\section{STRAIN-INDUCED AXIAL GAUGE FIELD}

We now discuss the physical origin of the axial gauge field. The basic idea is to incorporate lattice strain in the tightbinding model (1), which acts on the low-energy excitations as effective gauge fields. Strain shifts the lattice sites $\boldsymbol{R}$ by the displacement vector $\boldsymbol{u}(\boldsymbol{R})$, as $\boldsymbol{R}+\boldsymbol{u}(\boldsymbol{R})$, thereby modifying and introducing new overlaps between atomic orbitals. In our tight-binding model this changes the hopping parameters as $[51,52]$

$$
\begin{aligned}
t\left(\boldsymbol{a}_{x}\right) \tau_{z} & \simeq t\left(1-u_{x x}\right) \tau_{z}+i v u_{x z} \tau_{y}, \\
t\left(\boldsymbol{a}_{y}\right) \tau_{z} & \simeq t\left(1-u_{y y}\right) \tau_{z}+i v u_{y z} \tau_{y}, \\
t\left(\boldsymbol{a}_{z}\right) \tau_{z} & \simeq t\left(1-u_{z z}\right) \tau_{z}, \\
i v\left(\boldsymbol{a}_{z}\right) \tau_{y} & \simeq i v\left(1-u_{z z}\right) \tau_{y}+t \sum_{i \neq z} u_{z i} \tau_{z},
\end{aligned}
$$

where $t\left(\boldsymbol{a}_{\mu}\right) \quad(\mu=x, y, z)$ represents the hopping amplitudes along the bond direction $\boldsymbol{a}_{\mu}$ and $u_{\mu \nu}=\left[\partial_{\mu} u_{\nu}(\boldsymbol{R})+\right.$ $\left.\partial_{\nu} u_{\mu}(\boldsymbol{R})\right] / 2$ is the symmetrized strain tensor. The first terms in Eqs. (10) describe changes in the hopping amplitudes between two like orbitals, when the bond lengths are modified by strain. The second terms originate from new hopping processes between different orbitals, which are symmetry forbidden in the unstrained lattice. In the following, we focus on the gauge fields induced by the $u_{z v}$ components of the strain tensor, as these are the ones that probe the parity anomaly. The other components of $u_{\mu \nu}$ only renormalize the Fermi velocity, which is not important for our purpose. Using Eq. (10), we find that this lattice strain generates additional terms in the tight-binding Hamiltonian (1), $H(\boldsymbol{p}) \rightarrow H(\boldsymbol{p})+\delta H(\boldsymbol{p})$, which are of the form ${ }^{1}$

$$
\delta H(\boldsymbol{p}) \simeq-t u_{z z} \cos p_{z} a \tau_{z}+v\left(u_{x z} \sin p_{x} a+u_{y z} \sin p_{y} a\right) \tau_{y} .
$$

These modifications change the low-energy Hamiltonian (6) to

$$
\begin{aligned}
H_{\mathrm{eff}}(\boldsymbol{p})+\delta H_{\mathrm{eff}}(\boldsymbol{p}) \simeq & v_{F}\left(q_{r}-A_{r}^{5}\right) \tau_{z} \\
& +\lambda\left(p_{z}-A_{z}^{5}(\varphi)\right) \tau_{y}+\Delta \tau_{x},
\end{aligned}
$$

\footnotetext{
${ }^{1}$ Here we have also neglected the term $v u_{z z} \sin k_{z} \tau_{y}$, as it only renormalizes the hopping amplitude $v$.
}

with the pseudo-gauge-potentials

$$
\begin{aligned}
A_{r}^{5} & =u_{z z} / p_{0} a^{2}, \\
A_{z}^{5}(\varphi) & =\sum_{i} f_{i}(\varphi) A_{z}^{i, 5} \simeq-p_{0}\left(u_{x z} \cos \varphi+u_{y z} \sin \varphi\right)
\end{aligned}
$$

along the $r$ and $z$ directions, respectively, where $f_{x}(\varphi)=$ $p_{0} \cos \varphi, f_{y}(\varphi)=p_{0} \sin \varphi$, and $A_{z}^{i, 5}=-u_{i z}(i=x, y)$. Here we have introduced the Fermi velocity $v_{F}=p_{0} / m$, the radial momentum $q_{r}=p_{r}-p_{0}$, and the cylindrical coordinates $\left(p_{r}, p_{z}, \varphi\right)$ with $p_{r} \in[0, \infty), p_{z} \in(-\infty, \infty)$, and $\varphi \in$ $[0,2 \pi)$.

We conclude that in NLSMs with a nodal ring in the $p_{z}=0$ plane, the strain field components $u_{z v}$ act on the low-energy excitations like effective gauge potentials. Interestingly, these effective gauge potentials are axial, since they couple with opposite sign to the excitations at opposite sides of the nodal ring, i.e., at $\left(p_{0}, 0, \varphi\right)$ and $\left(p_{0}, 0, \varphi+\pi\right)$. From Eq. (12) we see that $A_{r}^{5}$ concentrically shrinks or expands the nodal ring [Fig. 2(a)], while $A_{z}^{i, 5}$ tilts the nodal ring out of the $p_{z}=0$ plane [Fig. 2(b)].

If we consider time-dependent lattice strain, i.e., lattice vibrations, we can also generate axial electric fields. That is, the time dependence of the strain tensor $u_{\mu z}(t)$ produces axial electric fields via

$$
E_{r}^{5}=-\frac{\partial A_{r}^{5}}{\partial t}, \quad E_{z}^{i, 5}=-\frac{\partial A_{z}^{i, 5}}{\partial t},
$$

where the axial electric fields $E_{z}^{i, 5}$ are defined by the angularindependent parts of the axial vector potentials and $f_{i}(\varphi)$ are absorbed into the axial charge coupling constants.

\section{TOPOLOGICAL PIEZOELECTRIC EFFECT}

Next, we demonstrate that axial electric fields in NLSMs induce net topological currents that flow in the direction perpendicular to the axial fields. For that purpose we use linear response theory to compute the axial conductivity tensors $\tilde{\sigma}_{\mu r}(\omega)$ and $\tilde{\sigma}_{\mu r}^{x}(\omega)$, which are defined as

$$
\left\langle\hat{j}_{\mu}\right\rangle(\omega)=\tilde{\sigma}_{\mu r}(\omega) E_{r}^{5}(\omega)+\tilde{\sigma}_{\mu z}^{x}(\omega) E_{z}^{x, 5}(\omega),
$$

with the current density operator $\hat{\boldsymbol{j}}$. By use of Kubo's formula, we compute the axial Hall conductivities $\tilde{\sigma}_{z r}(T, \omega)$ and $\tilde{\sigma}_{x z}(T, \omega)$. [See Appendix A for details of the derivation of Eqs. (15) and a discussion of the effects of spin-orbit coupling.] In the DC limit $\omega \rightarrow 0$, they are given by

$$
\begin{aligned}
\tilde{\sigma}_{z r}^{\mathrm{DC}}(T) & =-\frac{1}{V} \sum_{\boldsymbol{p}, \alpha} f\left(\epsilon_{\boldsymbol{p}}^{\alpha}\right) \mathcal{B}_{z r}^{\alpha}(\boldsymbol{p}), \\
\tilde{\sigma}_{x z}^{x, \mathrm{DC}}(T) & =-\frac{1}{V} \sum_{\boldsymbol{p}, \alpha} f\left(\epsilon_{\boldsymbol{p}}^{\alpha}\right) p_{0} \cos \varphi \mathcal{B}_{x z}^{\alpha}(\boldsymbol{p}),
\end{aligned}
$$

where $\epsilon_{\boldsymbol{p}}^{\alpha}, f\left(\epsilon_{\boldsymbol{p}}^{\alpha}\right)$, and $\mathcal{B}_{\mu \nu}^{\alpha}(\boldsymbol{p})=-2 \operatorname{Im}\left\langle\partial_{p_{\mu}} u_{\alpha}(\boldsymbol{p}) \mid \partial_{p_{\nu}} u_{\alpha}(\boldsymbol{p})\right\rangle$ are the energy of the Bloch electrons, the Fermi function, and the Berry curvature, respectively.

Thus, it follows that axial electric fields produce transverse Hall currents, whose magnitude is determined by the Berry 
(a)

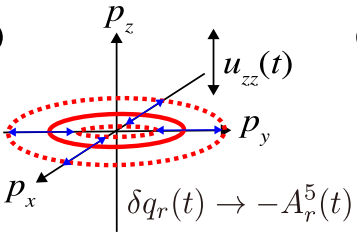

(b)

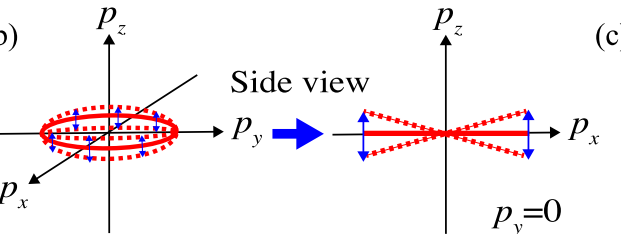

(c)

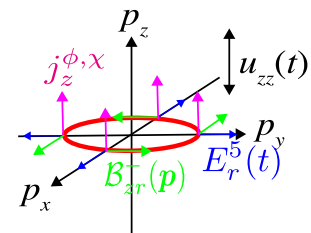

(d)

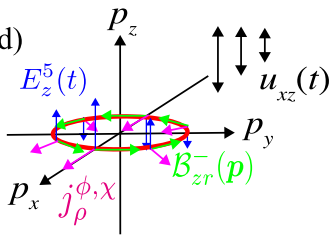

FIG. 2. (a) and (b) The axial gauge potential $A_{r}^{5}(t)$ changes the size of the nodal ring, while $A_{z}^{5}(t)$ tilts it out of plane, as indicated by the dashed lines. (c) and (d) The strain-induced currents $\boldsymbol{j}^{\phi, \chi}$ (pink) are perpendicular to both the Berry curvature $\mathcal{B}_{z r}^{-}(\boldsymbol{p})$ (green) and the axial electric field $\boldsymbol{E}^{5}$ (blue).

curvature. These Hall currents are perpendicular to both the axial electric field and the Berry curvature [see Figs. 2(c) and 2(d)]. For instance, axial electric fields along the $r$ direction lead to electric currents in the $z$ direction, since the direction of the Berry curvature is within the $p_{z}=0$ plane. Similarly, axial electric fields along the $z$ direction produce currents in the $p_{z}=0$ plane. Because the axial electric fields are generated by lattice vibrations, we refer to this type of Hall response as a topological piezoelectric effect.

Interestingly, in the low-frequency regime $|\omega / \Delta| \ll 1$, the axial Hall conductivities become semiquantized, i.e., their magnitude depends only on the length of the nodal $\operatorname{ring} L=$ $2 \pi p_{0}$. That is, in the limit $|\omega / \Delta| \ll 1$ we find

$$
\begin{gathered}
\tilde{\sigma}_{z r}^{\mathrm{DC}}(T=0) \simeq-\frac{L}{8 \pi^{2}} \operatorname{sign}(\Delta), \\
\tilde{\sigma}_{x z}^{x, \mathrm{DC}}(T=0) \simeq \frac{L}{16 \pi^{2}} \operatorname{sign}(\Delta),
\end{gathered}
$$

where $\left|\Delta /\left(v_{F} p_{\text {cut }}\right)\right|,\left|\Delta /\left(\lambda p_{\text {cut }}\right)\right| \ll 1$ is assumed, with some cutoff momentum $p_{\text {cut }}$. This is confirmed by numerical evaluations of $\tilde{\sigma}_{z r}(T=0, \omega)$ (see Fig. 3). We observe in Figs. 3(a) and 3(b) that the axial Hall conductivity asymptotically approaches its semiquantized value for $\omega \rightarrow 0$, once $\Delta$ becomes sufficiently small and $p_{\text {cut }}$ sufficiently large, respectively. As displayed in the inset of Fig. 3(a), the semiquantized value of the DC axial Hall conductivity scales linearly with the size of the nodal ring.
Before concluding, we show that the TPEE is related to the polarization current of NLSMs. As discussed above, the axial electric field $E_{r}^{5}(t)$ periodically shrinks and expands the nodal ring. This leads to a periodic fluctuation of the bulk electric polarization, which is determined by the size of the nodal ring. Hence, the axial electric field generates a polarization current, which according to Eq. (5) takes the form

$$
j_{z}^{\mathrm{pol}}=\frac{d P_{z}(t)}{d t}=\frac{1}{8 \pi^{2}} \frac{d S(t)}{d t} \simeq-\frac{L}{8 \pi^{2}} \operatorname{sign}(\Delta) E_{r}^{5}(t),
$$

where $S(t)=\pi\left[p_{0}+A_{r}^{5}(t)\right]^{2}$ is the area of the nodal ring. Since Eq. (17) coincides with Eq. (16b), we conclude that the TPEE is linked to the polarization current of NLSMs and that its quantization arises from the semiquantized electric polarization.

The proposed TPEE is testable in the materials CaAgP or $\mathrm{Ca}_{3} \mathrm{P}_{2}$, which possess a single nodal ring near the Fermi energy (see Fig. 4). In these materials lattice vibrations can be generated by sound waves, which leads to an AC via the TPEE [53,54]. Radiating an ultrasonic wave along the $z$ direction generates a displacement vector of the form

$$
u_{z}(t, z)=u_{z} \cos \left(q_{z} z-\omega t\right),
$$

where $q_{z}$ is the wave vector and $\omega$ is the frequency of the acoustic wave. From Eq. (18) the amplitude of the strain tensor is roughly given by $\sim u_{z} q_{z} /(2 \pi)$. To estimate the typical magnitude of the TPEE in the case of $\mathrm{Ca}_{3} \mathrm{P}_{2}$, we consider an ultrasonic wave with the frequency $\omega=100 \mathrm{MHz}$,
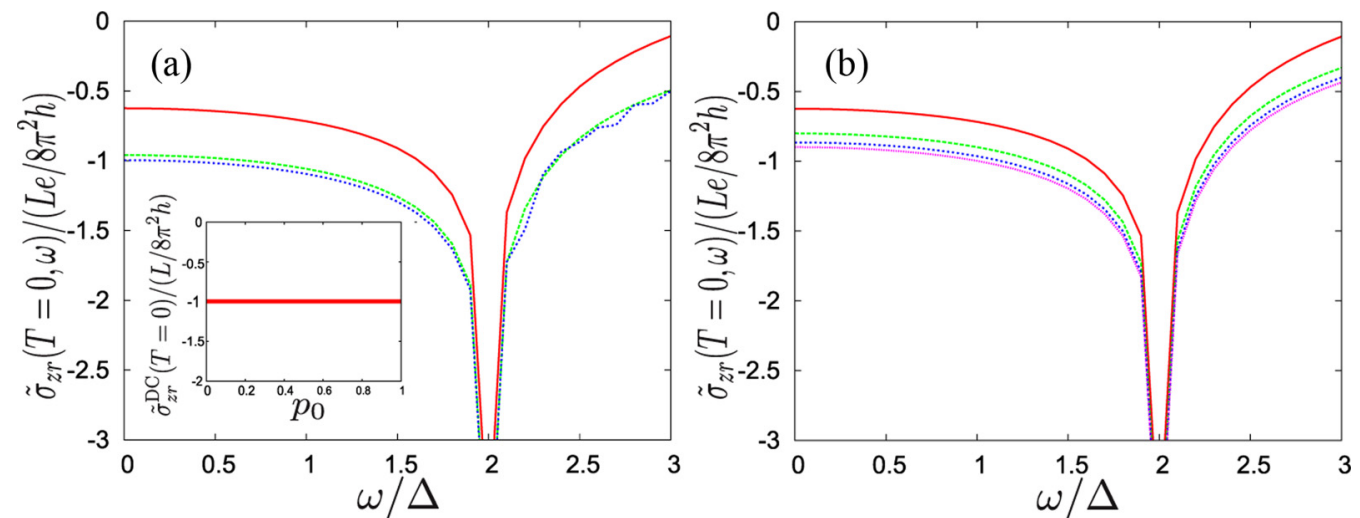

FIG. 3. Frequency dependence of the axial Hall conductivity $\tilde{\sigma}_{z r}(T=0, \omega)$ for different values of $\Delta$ and $p_{\text {cut }}$. In (a) the red, green, and blue curves correspond to $\Delta /\left(v_{F} p_{0}\right)=0.1,0.01$, and 0.001 , respectively, with $p_{\text {cut }}$ fixed at $p_{\text {cut }}=0.2 p_{0}$. In (b) the red, green, blue, and pink curves correspond to $p_{\text {cut }} /\left(p_{0} / 10\right)=2,4,6$, and 8 , respectively, with $\Delta$ fixed at $\Delta=0.1 v_{F} p_{0}$. The inset shows the $p_{0}$ dependence of the $\mathrm{DC}$ axial Hall conductivity $\tilde{\sigma}_{z r}^{\mathrm{DC}}(T=0)$ with $p_{\text {cut }}=0.2 p_{0}$ and $\Delta=0.0004$. The other parameters in all panels are $v_{F}=2.0, p_{0}=0.2$, and $\lambda=2.5$. 


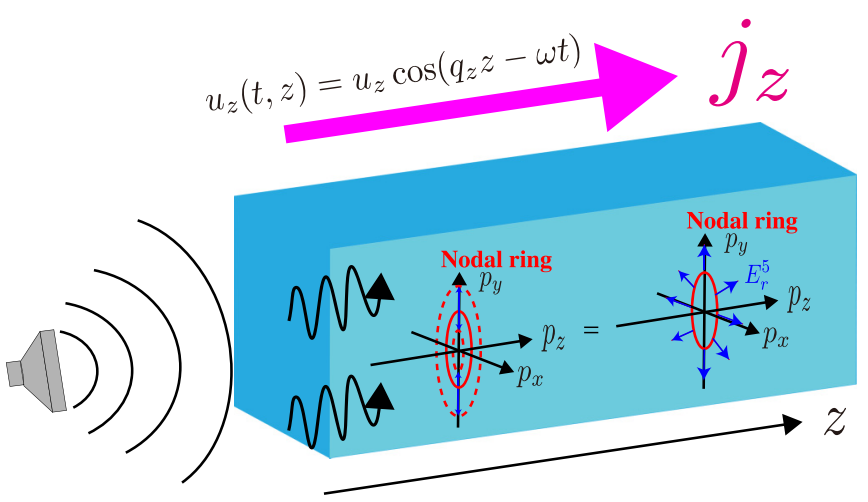

acoustic wave radiation

FIG. 4. Schematic of the experimental setup for the detection of the TPEE induced by ultrasonic wave radiation.

$q_{z}=2.0 \times 10^{4} \mathrm{~m}^{-1}$, and sound velocity $\sim 5000 \mathrm{~m} / \mathrm{s}$. Further, we assume that $u_{z}=5.0 \AA$ and the parity-breaking term is about $\left|\Delta /\left(v_{F} p_{0}\right)\right|=1.0 \times 10^{-3}$. The relevant material parameters for $\mathrm{Ca}_{3} \mathrm{P}_{2}$ are $v_{F}=2.72 \times 10^{5} \mathrm{~m} / \mathrm{s}, \lambda=3.80 \times$ $10^{5} \mathrm{~m} / \mathrm{s}, p_{0}=0.206 \AA^{-1}$, and $a=8.26 \AA$ [43]. From this we estimate the TPEE current to be about $j_{z} \simeq 1.9 \mathrm{~mA} / \mathrm{cm}^{2}$, which is experimentally detectable. ${ }^{2}$

\section{CONCLUSION}

We have shown that dynamical strain induces a TPEE in NLSMs, which manifests itself by dissipationless Hall currents, originating from the parity anomaly. We have focused on NLSMs with negligible spin-orbit coupling (SOC); the case of strong SOC is briefly discussed in Appendix B. While it would be of fundamental interest to observe the parity anomaly in NLSMs using sound waves, the TPEE could also be useful for future piezoelectric devices, e.g., sound wave or vibration detectors. Since NLSMs can be easily switched between a semimetallic regime with high mobility and a piezoelectric regime using, e.g., strain, they could also be used for controllable multifunctional devices. Finally, we note that the concept of the TPEE can be extended straightforwardly to two-dimensional Dirac semimetals.

\section{ACKNOWLEDGMENTS}

T.M. thanks K. Nomura, A. Yamakage, and Y. Ominato for invaluable discussion. T.M. was supported by a JSPS Fellowship for Young Scientists. S.F. was supported by the Grant-in-Aid for Scientific Research from JSPS of Japan (Grant No. 17K05517), KAKENHI on Innovative Areas "Topological Materials Science" (Grant No. JP15H05852), "J-Physics" (Grant No. JP18H04318), and JST CREST Grant No. JPMJCR19T5, Japan. This research was initiated at KITP UC Santa Barbara and supported in part by the National Science Foundation under Grant No. NSF PHY-1748958.

\footnotetext{
${ }^{2}$ As verified in Refs. [36,43], the spin-orbit coupling in $\mathrm{CaAgP}$ and $\mathrm{Ca}_{3} \mathrm{P}_{2}$ is negligible. See also Appendix $\mathrm{B}$ for the effects of the spinorbit coupling on the TPEE.
}

\section{APPENDIX A: DERIVATION OF EQ. (15)}

To derive the axial Hall conductivities $\tilde{\sigma}_{z r}$ and $\tilde{\sigma}_{x z}^{x}$ we use the Bloch eigenbasis $\left\{\left|u_{ \pm}(\boldsymbol{p})\right\rangle\right\}$ with

$$
\begin{aligned}
H_{\mathrm{eff}}(\boldsymbol{p})\left|u_{ \pm}(\boldsymbol{p})\right\rangle & =\epsilon_{\boldsymbol{p}}^{ \pm}\left|u_{ \pm}(\boldsymbol{p})\right\rangle, \\
\epsilon_{\boldsymbol{p}}^{ \pm} & = \pm \sqrt{v_{F}^{2} q_{r}^{2}+\lambda^{2} p_{z}^{2}+\Delta^{2}}
\end{aligned}
$$

and express the current density operator as

$$
\hat{\boldsymbol{j}}=\sum_{\boldsymbol{p}, \alpha= \pm} \frac{\partial \epsilon_{\boldsymbol{p}}^{\alpha}}{\partial \boldsymbol{p}} \hat{c}_{\boldsymbol{p} \alpha}^{\dagger} \hat{c}_{\boldsymbol{p} \alpha}+i \sum_{\alpha \neq \beta}\left(\epsilon_{\boldsymbol{p}}^{\alpha}-\epsilon_{\boldsymbol{p}}^{\beta}\right) \boldsymbol{A}^{\alpha, \beta}(\boldsymbol{p}) \hat{c}_{\boldsymbol{p} \alpha}^{\dagger} \hat{c}_{\boldsymbol{p} \beta},
$$

where $\hat{c}_{\boldsymbol{p} \alpha}^{\dagger}$ and $\hat{c}_{\boldsymbol{p} \alpha}$ are creation and annihilation operators, respectively. From this expression and with the use of the Kubo formula we obtain the axial Hall conductivities

$$
\begin{aligned}
& \tilde{\sigma}_{z r}(T, \omega)=-\lim _{\delta \rightarrow+0} \frac{i}{V} \sum_{p, \alpha \neq \beta} f\left(\epsilon_{p}^{\alpha}\right)\left(\epsilon_{p}^{\alpha}-\epsilon_{p}^{\beta}\right) \\
& \times\left[\frac{\mathcal{A}_{z}^{\alpha, \beta} \mathcal{A}_{r}^{\beta, \alpha}}{\omega+\epsilon_{p}^{\alpha}-\epsilon_{p}^{\beta}+i \delta}+\frac{\mathcal{A}_{r}^{\alpha, \beta} \mathcal{A}_{z}^{\beta, \alpha}}{\omega-\epsilon_{p}^{\alpha}+\epsilon_{p}^{\beta}+i \delta}\right], \\
& \tilde{\sigma}_{x z}^{x}(T, \omega)=-\lim _{\delta \rightarrow+0} \frac{i}{V} \sum_{\boldsymbol{p}, \alpha \neq \beta} f\left(\epsilon_{\boldsymbol{p}}^{\alpha}\right) p_{0} \cos \varphi\left(\epsilon_{\boldsymbol{p}}^{\alpha}-\epsilon_{\boldsymbol{p}}^{\beta}\right) \\
& \times\left[\frac{\mathcal{A}_{x}^{\alpha, \beta} \mathcal{A}_{z}^{\beta, \alpha}}{\omega+\epsilon_{\boldsymbol{p}}^{\alpha}-\epsilon_{\boldsymbol{p}}^{\beta}+i \delta}+\frac{\mathcal{A}_{z}^{\alpha, \beta} \mathcal{A}_{x}^{\beta, \alpha}}{\omega-\epsilon_{\boldsymbol{p}}^{\alpha}+\epsilon_{\boldsymbol{p}}^{\beta}+i \delta}\right] .
\end{aligned}
$$

We note that the factor $p_{0} \cos \varphi$ in Eq. (A3b) originates from the momentum dependence of the axial electric field. In the DC limit $\omega \rightarrow 0$, we obtain Eqs. (15), where $\mathcal{B}_{\mu \nu}^{\alpha}(\boldsymbol{p})=$ $-2 \operatorname{Im}\left\langle\partial_{p_{\mu}} u_{\alpha}(\boldsymbol{p}) \mid \partial_{p_{v}} u_{\alpha}(\boldsymbol{p})\right\rangle$ is the Berry curvature, which is given by

$$
\begin{aligned}
& \mathcal{B}_{z r}^{ \pm}(\boldsymbol{p})=\mp \frac{\Delta \lambda v_{F}}{2\left(v_{F}^{2} q_{r}^{2}+\lambda^{2} p_{z}^{2}+\Delta^{2}\right)^{3 / 2}}, \\
& \mathcal{B}_{x z}^{ \pm}(\boldsymbol{p})= \pm \frac{\Delta \lambda v_{F} \cos \varphi}{2\left(v_{F}^{2} q_{r}^{2}+\lambda^{2} p_{z}^{2}+\Delta^{2}\right)^{3 / 2}}
\end{aligned}
$$

\section{APPENDIX B: EFFECTS OF SPIN-ORBIT COUPLING}

In the main text we considered materials with very weak SOC, such as $\mathrm{Ca}_{3} \mathrm{P}_{2}$ and $\mathrm{CaAgP}$, where the topological properties are protected by $\mathrm{SU}(2)$ spin-rotation symmetry in combination with reflection symmetry and/or $\mathcal{P} \mathcal{T}$ symmetry. In these materials SOC is at least three orders of magnitude smaller than the bandwidth. Such small SOC slightly breaks the $\mathrm{SU}(2)$ spin-rotation symmetry and possibly also the reflection symmetry, which opens a very small gap at the nodal line. Nevertheless, such small SOC does not substantially alter the Berry curvature carried by the bands. As a result the transverse topological currents and the TPEE remain largely unchanged; only their magnitude is slightly reduced and the semiquantization slightly broken [43]. In $\mathrm{Ca}_{3} \mathrm{P}_{2}$ and $\mathrm{CaAgP}$ SOC is 
(a)

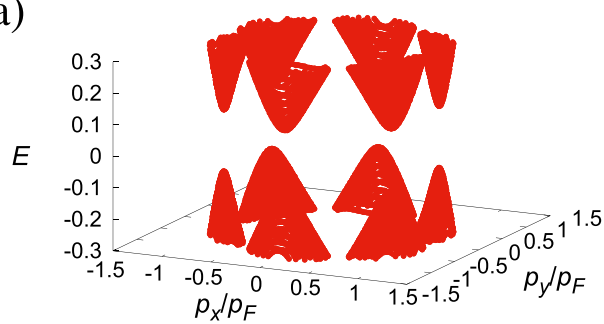

(b)

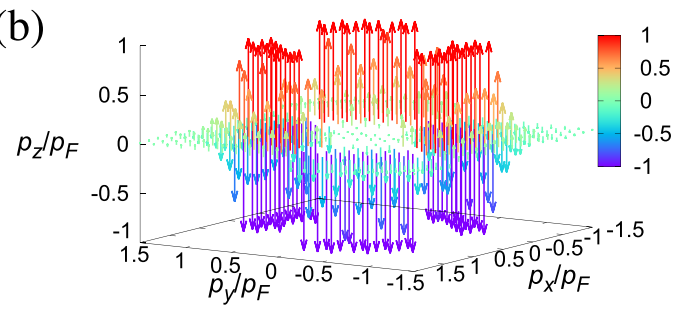

FIG. 5. (a) Energy dispersion near the nodal line of CaAgAs. Spin-orbit coupling opens a gap of about 20 meV. (b) Berry curvature of the occupied states of CaAgAs in the presence of SOC.

so small that these effects can be neglected. Hence, these materials are good platforms for the experimental verification of the TPEE.

However, there are also nodal-line materials with strong SOC, which fully breaks the SU(2) spin-rotation symmetry. This either splits the Dirac nodal line into two Weyl nodal lines or leads to a full gap in the spectrum. The former occurs in $\mathrm{PbTaSe}_{2}$ and $\mathrm{TlTaSe}_{2}$, which exhibit Weyl nodal lines that are protected by reflection symmetry and time-reversal symmetry $[40,43,55]$. The topology of these two materials is characterized by a reflection invariant that guarantees the stability of the nodal line. Moreover, these two materials exhibit a reflection anomaly, which leads to a similar topological response and TPEE as in $\mathrm{CaAgAs}$ and $\mathrm{Ca}_{3} \mathrm{P}_{2}$.

An example of a nodal-line material where SOC generates a large gap is CaAgAs. In the absence of SOC, this material has a nodal ring within the $p_{z}=0$ plane, which is protected by mirror-reflection symmetry. The SOC in CaAgAs breaks not only SU(2) spin-rotation symmetry, but also the mirrorreflection symmetry, which opens up an energy gap of about $20 \mathrm{meV}$. This transforms the nodal-line semimetal into a topological insulator with a $\mathbb{Z}_{2}$ invariant [36]. We will now study whether the presence of an SOC-induced gap of $20 \mathrm{meV}$ removes the TPEE in CaAgAs or not. For this purpose we consider the effective continuum Hamiltonian [36]

$$
H_{\mathrm{CaAgAs}}(\boldsymbol{p})=\left(\begin{array}{cc}
h(\boldsymbol{p}) & \Lambda(\boldsymbol{p}) \\
\Lambda^{\dagger}(\boldsymbol{p}) & h^{*}(-\boldsymbol{p})
\end{array}\right),
$$

where

$$
\begin{gathered}
h(\boldsymbol{p})=\frac{\boldsymbol{p}^{2}-p_{F}^{2}}{2 m} \sigma_{z}+A p_{z}\left(p_{x} \sigma_{x}-p_{y} \sigma_{y}\right), \\
\Lambda(\boldsymbol{p})=-i B_{\perp} p_{z}\left(1-\sigma_{z}\right)-i B_{\|}\left(p_{x}+i p_{y}\right) \sigma_{x}-i D\left(p_{x}-i p_{y}\right)^{2} \sigma_{y} .
\end{gathered}
$$

Equation (B3) represent an asymmetric SOC which breaks with its strong momentum dependence the mirror-reflection symmetry. As a consequence, a stronglymomentum-dependent gap is opened [see Fig. 5(a)]. The strongly-momentum-dependent SOC leads to a Berry curvature profile, which is qualitatively different from that of $\mathrm{Ca}_{3} \mathrm{P}_{2}$ [compare Fig. 5(b) with Fig. 2]. If an axial electric field is applied along the $r$ direction, the topological Hall current in $\mathrm{CaAgAs}$ therefore cancels out. Hence, the TPEE in CaAgAs is destroyed by the asymmetric SOC. This is in agreement with the fact that $\mathrm{CaAgAs}$ does not exhibit a semiquantized bulk electric polarization. The key for the realization of a TPEE is a semiquantized bulk electric polarization. If a large enough SOC breaks it, then the TPEE is not realized.
[1] P. Hosur and X. Qi, Recent developments in transport phenomena in Weyl semimetals, C. R. Phys. 14, 857 (2013).

[2] N. P. Armitage, E. J. Mele, and A. Vishwanath, Weyl and Dirac semimetals in three-dimensional solids, Rev. Mod. Phys. 90, 015001 (2018).

[3] T. O. Wehling, A. M. Black-Schaffer, and A. V. Balatsky, Dirac materials, Adv. Phys. 63, 1 (2014).

[4] S. Murakami, Phase transition between the quantum spin Hall and insulator phases in 3D: Emergence of a topological gapless phase, New J. Phys. 9, 356 (2007).

[5] X. Wan, A. M. Turner, A. Vishwanath, and S. Y. Savrasov, Topological semimetal and Fermi-arc surface states in the electronic structure of pyrochlore iridates, Phys. Rev. B 83, 205101 (2011).

[6] A. A. Burkov, M. D. Hook, and L. Balents, Topological nodal semimetals, Phys. Rev. B 84, 235126 (2011).

[7] K. Nakada, M. Fujita, G. Dresselhaus, and M. S. Dresselhaus, Edge state in graphene ribbons: Nanometer size effect and edge shape dependence, Phys. Rev. B 54, 17954 (1996).
[8] A. N. Redlich, Parity violation and gauge noninvariance of the effective gauge field action in three dimensions, Phys. Rev. D 29, 2366 (1984).

[9] F. D. M. Haldane, Model for a Quantum Hall Effect without Landau Levels: Condensed-Matter Realization of the Parity Anomaly, Phys. Rev. Lett. 61, 2015 (1988).

[10] H. Nielsen and M. Ninomiya, The Adler-Bell-Jackiw anomaly and Weyl fermions in a crystal, Phys. Lett. B 130, 389 (1983).

[11] A. A. Zyuzin and A. A. Burkov, Topological response in Weyl semimetals and the chiral anomaly, Phys. Rev. B 86, 115133 (2012).

[12] P. Goswami and S. Tewari, Axionic field theory of $(3+1)$ dimensional Weyl semimetals, Phys. Rev. B 88, 245107 (2013).

[13] D. T. Son and B. Z. Spivak, Chiral anomaly and classical negative magnetoresistance of Weyl metals, Phys. Rev. B 88, 104412 (2013).

[14] A. A. Burkov and Y. B. Kim, $\mathbb{Z}_{2}$ and Chiral Anomalies in Topological Dirac Semimetals, Phys. Rev. Lett. 117, 136602 (2016). 
[15] K.-Y. Yang, Y.-M. Lu, and Y. Ran, Quantum Hall effects in a Weyl semimetal: Possible application in pyrochlore iridates, Phys. Rev. B 84, 075129 (2011).

[16] K. Fukushima, D. E. Kharzeev, and H. J. Warringa, Chiral magnetic effect, Phys. Rev. D 78, 074033 (2008).

[17] M. M. Vazifeh and M. Franz, Electromagnetic Response of Weyl Semimetals, Phys. Rev. Lett. 111, 027201 (2013).

[18] P. Goswami, J. H. Pixley, and S. Das Sarma, Axial anomaly and longitudinal magnetoresistance of a generic three-dimensional metal, Phys. Rev. B 92, 075205 (2015).

[19] X. Huang, L. Zhao, Y. Long, P. Wang, D. Chen, Z. Yang, H. Liang, M. Xue, H. Weng, Z. Fang, X. Dai, and G. Chen, Observation of the Chiral-Anomaly-Induced Negative Magnetoresistance in 3D Weyl Semimetal TaAs, Phys. Rev. X 5, 031023 (2015).

[20] J. Xiong, S. K. Kushwaha, T. Liang, J. W. Krizan, M. Hirschberger, W. Wang, R. J. Cava, and N. P. Ong, Evidence for the chiral anomaly in the Dirac semimetal $\mathrm{Na}_{3} \mathrm{Bi}$, Science 350, 413 (2015).

[21] R. Ilan, A. G. Grushin, and D. I. Pikulin, Pseudoelectromagnetic fields in 3D topological semimetals, Nat. Rev. Phys. 2, 29 (2020).

[22] F. Guinea, M. Katsnelson, and A. Geim, Energy gaps and a zero-field quantum Hall effect in graphene by strain engineering, Nat. Phys. 6, 30 (2010).

[23] N. Levy, S. Burke, K. Meaker, M. Panlasigui, A. Zettl, F. Guinea, A. C. Neto, and M. Crommie, Strain-induced pseudomagnetic fields greater than 300 tesla in graphene nanobubbles, Science 329, 544 (2010).

[24] P. Nigge, A. C. Qu, É. Lantagne-Hurtubise, E. Mårsell, S. Link, G. Tom, M. Zonno, M. Michiardi, M. Schneider, S. Zhdanovich, G. Levy, U. Starke, C. Gutiérrez, D. Bonn, S. A. Burke, M. Franz, and A. Damascelli, Room temperature straininduced Landau levels in graphene on a wafer-scale platform, Sci. Adv. 5, eaaw5593 (2019).

[25] C.-X. Liu, P. Ye, and X.-L. Qi, Chiral gauge field and axial anomaly in a Weyl semimetal, Phys. Rev. B 87, 235306 (2013).

[26] A. G. Grushin, J. W. F. Venderbos, A. Vishwanath, and R. Ilan, Inhomogeneous Weyl and Dirac Semimetals: Transport in Axial Magnetic Fields and Fermi Arc Surface States from PseudoLandau Levels, Phys. Rev. X 6, 041046 (2016).

[27] T. L. Hughes, R. G. Leigh, and O. Parrikar, Torsional anomalies, Hall viscosity, and bulk-boundary correspondence in topological states, Phys. Rev. D 88, 025040 (2013).

[28] O. Parrikar, T. L. Hughes, and R. G. Leigh, Torsion, parity-odd response, and anomalies in topological states, Phys. Rev. D 90, 105004 (2014).

[29] H. Sumiyoshi and S. Fujimoto, Torsional Chiral Magnetic Effect in a Weyl Semimetal with a Topological Defect, Phys. Rev. Lett. 116, 166601 (2016).

[30] D. I. Pikulin, A. Chen, and M. Franz, Chiral Anomaly from Strain-Induced Gauge Fields in Dirac and Weyl Semimetals, Phys. Rev. X 6, 041021 (2016).

[31] T. Kobayashi, T. Matsushita, T. Mizushima, A. Tsuruta, and S. Fujimoto, Negative Thermal Magnetoresistivity as a Signature of a Chiral Anomaly in Weyl Superconductors, Phys. Rev. Lett. 121, 207002 (2018).

[32] Y. Ishihara, T. Mizushima, A. Tsuruta, and S. Fujimoto, Torsional chiral magnetic effect due to skyrmion textures in a Weyl superfluid ${ }^{3} \mathrm{He}$-A, Phys. Rev. B 99, 024513 (2019).
[33] T. Matsushita, T. Liu, T. Mizushima, and S. Fujimoto, Charge/spin supercurrent and the Fulde-Ferrell state induced by crystal deformation in Weyl/Dirac superconductors, Phys. Rev. B 97, 134519 (2018).

[34] D. Kurebayashi and K. Nomura, Theory for spin torque in Weyl semimetal with magnetic texture, Sci. Rep. 9, 5365 (2019).

[35] S.-Y. Yang, H. Yang, E. Derunova, S. S. P. Parkin, B. Yan, and M. N. Ali, Symmetry demanded topological nodal-line materials, Adv. Phys. X 3, 1414631 (2018).

[36] A. Yamakage, Y. Yamakawa, Y. Tanaka, and Y. Okamoto, Line-node Dirac semimetal and topological insulating phase in noncentrosymmetric pnictides $\mathrm{CaAg} X(X=\mathrm{P}, \mathrm{As})$, J. Phys. Soc. Jpn 85, 013708 (2015).

[37] M. Neupane, I. Belopolski, M. M. Hosen, D. S. Sanchez, R. Sankar, M. Szlawska, S.-Y. Xu, K. Dimitri, N. Dhakal, P. Maldonado, P. M. Oppeneer, D. Kaczorowski, F. Chou, M. Z. Hasan, and T. Durakiewicz, Observation of topological nodal fermion semimetal phase in ZrSiS, Phys. Rev. B 93, 201104(R) (2016).

[38] D. Takane, K. Nakayama, S. Souma, T. Wada, Y. Okamoto, K. Takenaka, Y. Yamakawa, A. Yamakage, T. Mitsuhashi, K. Horiba et al., Observation of Dirac-like energy band and ring-torus Fermi surface associated with the nodal line in topological insulator CaAgAs, Quantum Mater. 3, 1 (2018).

[39] N. Xu, Y. T. Qian, Q. S. Wu, G. Autès, C. E. Matt, B. Q. Lv, M. Y. Yao, V. N. Strocov, E. Pomjakushina, K. Conder, N. C. Plumb, M. Radovic, O. V. Yazyev, T. Qian, H. Ding, J. Mesot, and M. Shi, Trivial topological phase of $\mathrm{CaAgP}$ and the topological nodal-line transition in $\mathrm{CaAgP}_{1-x} \mathrm{As}_{x}$, Phys. Rev. B 97, 161111(R) (2018).

[40] G. Bian, T.-R. Chang, R. Sankar, S.-Y. Xu, H. Zheng, T. Neupert, C.-K. Chiu, S.-M. Huang, G. Chang, I. Belopolski et al., Topological nodal-line fermions in spin-orbit metal $\mathrm{PbTaSe}_{2}$, Nat. Commun. 7, 10556 (2016).

[41] B. Feng, B. Fu, S. Kasamatsu, S. Ito, P. Cheng, C.-C. Liu, Y. Feng, S. Wu, S. K. Mahatha, P. Sheverdyaeva et al., Experimental realization of two-dimensional Dirac nodal line fermions in monolayer $\mathrm{Cu}_{2} \mathrm{Si}$, Nat. Commun. 8, 1007 (2017).

[42] Y. Ominato, A. Yamakage, and K. Nomura, Line-node Dirac semimetal and topological insulating phase in noncentrosymmetric pnictides $\operatorname{CaAg} X(X=\mathrm{P}, \mathrm{As})$, J. Phys. Soc. Jpn 88, 114701 (2019).

[43] Y.-H. Chan, C.-K. Chiu, M. Y. Chou, and A. P. Schnyder, $\mathrm{Ca}_{3} \mathrm{P}_{2}$ and other topological semimetals with line nodes and drumhead surface states, Phys. Rev. B 93, 205132 (2016).

[44] C. K. Chiu, Y. H. Chan, and A. P. Schnyder, Quantized Berry phase and surface states under reflection symmetry or spacetime inversion symmetry, arXiv:1810.04094.

[45] A. Sekine and N. Nagaosa, Tunable charged domain wall from topological confinement in nodal-line semimetals, Phys. Rev. B 101, 081102(R) (2020).

[46] S. T. Ramamurthy and T. L. Hughes, Quasitopological electromagnetic response of line-node semimetals, Phys. Rev. B 95, 075138 (2017).

[47] W. B. Rui, Y. X. Zhao, and A. P. Schnyder, Topological transport in Dirac nodal-line semimetals, Phys. Rev. B 97, 161113(R) (2018).

[48] Y. X. Zhao, A. P. Schnyder, and Z. D. Wang, Unified Theory of $\mathcal{P} \mathcal{T}$ and $\mathcal{C P}$ Invariant Topological Metals and Nodal Superconductors, Phys. Rev. Lett. 116, 156402 (2016). 
[49] D. Xiao, M.-C. Chang, and Q. Niu, Berry phase effects on electronic properties, Rev. Mod. Phys. 82, 1959 (2010).

[50] R. D. King-Smith and D. Vanderbilt, Theory of polarization of crystalline solids, Phys. Rev. B 47, 1651 (1993).

[51] A. Cortijo, Y. Ferreirós, K. Landsteiner, and M. A. H. Vozmediano, Elastic Gauge Fields in Weyl Semimetals, Phys. Rev. Lett. 115, 177202 (2015).

[52] H. Shapourian, T. L. Hughes, and S. Ryu, Viscoelastic response of topological tight-binding models in two and three dimensions, Phys. Rev. B 92, 165131 (2015).
[53] P. O. Sukhachov and H. Rostami, Acoustogalvanic Effect in Dirac and Weyl Semimetals, Phys. Rev. Lett. 124, 126602 (2020).

[54] Y. Ferreiros, Y. Kedem, E. J. Bergholtz, and J. H. Bardarson, Mixed Axial-Torsional Anomaly in Weyl Semimetals, Phys. Rev. Lett. 122, 056601 (2019).

[55] G. Bian, T.-R. Chang, H. Zheng, S. Velury, S.-Y. Xu, T. Neupert, C.-K. Chiu, S.-M. Huang, D. S. Sanchez, I. Belopolski, N. Alidoust, P.-J. Chen, G. Chang, A. Bansil, H.-T. Jeng, H. Lin, and M. Z. Hasan, Drumhead surface states and topological nodal-line fermions in $\mathrm{TlTaSe}_{2}$, Phys. Rev. B 93, 121113(R) (2016). 